\title{
Non-receptor protein-tyrosine kinases as molecular targets for antiangiogenic therapy (Review)
}

\author{
SHIGERU KANDA ${ }^{1,3}$, YASUYOSHI MIYATA ${ }^{2}$, HIROSHI KANETAKE ${ }^{2}$ and THOMAS E. SMITHGALL ${ }^{4}$ \\ ${ }^{1}$ Department of Molecular Microbiology and Immunology, Division of Endothelial Cell Biology, and ${ }^{2}$ Department \\ of Urology, Nagasaki University Graduate School of Biomedical Sciences, 1-7-1 Sakamoto, Nagasaki 852-8501; \\ ${ }^{3}$ Department of Experimental and Clinical Laboratory Medicine, National Hospital Organization, Nagasaki Hospital, \\ 41-6 Sakuragi-machi, Nagasaki 851-0251, Japan; ${ }^{4}$ Department of Molecular Genetics and Biochemistry, \\ University of Pittsburgh School of Medicine, E1240 Biomedical Science Tower, Pittsburgh, PA 15261, USA
}

Received March 2, 2007; Accepted April 5, 2007

\begin{abstract}
Antiangiogenic therapy, including blockade of vascular endothelial growth factor (VEGF) signaling, was highly anticipated to improve the prognosis for patients with advanced cancers following the success of preclinical animal models. However, antiangiogenic monotherapy with VEGF antagonists has produced disappointing results in clinical trials to date. One of the reasons for this poor outcome is that angiogenesis is not solely regulated by VEGF. Inhibition of VEGF signaling, therefore, may select for tumor cell populations that stimulate angiogenesis through VEGF-independent pathways. Successful antiangiogenic therapy, therefore, may require simultaneous blockade of signaling downstream from multiple proangiogenic factor receptors. Recently, we found that nonreceptor protein-tyrosine kinases, including members of the Src and Fes families, play vital roles in the responses of cultured endothelial cells to several proangiogenic factors. In this review, we summarize the contributions of these kinase families to angiogenic pathways in endothelial cells, and discuss the potential of these kinases as new targets for antiangiogenic drug discovery.
\end{abstract}

\section{Contents}

1. Introduction

2. Roles of Src family kinases in angiogenesis

3. Roles of Fes in angiogenesis

Correspondence to: Dr Shigeru Kanda, National Hospital Organization, Nagasaki Hospital, 41-6 Sakuragi-machi, Nagasaki 851-0251, Japan

E-mail: skanda-jua@umin.net

Key words: angiogenesis inhibition, Src, Fes, endothelial cell, differentiation, migration

\section{Introduction}

Evidence for the dependence of solid tumor growth on angiogenesis has been provided by several animal models (1-4). Growth of tumors larger than 2-3 $\mathrm{mm}$ in diameter requires neo-vascularization to supply oxygen and nutrients (5). Once new blood vessels are established, tumors rapidly grow, invade surrounding interstitial tissue, and form distant metastasis. Many reports have demonstrated that the inhibition of angiogenesis, particularly sole inhibition of vascular endothelial growth factor (VEGF) signaling, significantly reduced the growth of tumor xenografts in rodents (6). However, suppression of VEGF signaling alone has failed to cause tumor regression or improve patient survival in clinical trials $(7,8)$. How can this remarkable difference in efficacy of antiangiogenic therapy in rodents and humans be explained? Possibilities include: i) antiangiogenic therapy targeting a single class of proangiogenic factor may select for tumor cell populations that induce angiogenesis through VEGF-independent mechanisms; ii) human tumors and xenografted cancers in animal models are differentially sensitive to hypoxia; and iii) normal endothelial cells display differential sensitivity to antiangiogenic molecules compared to tumor endothelial cells. Each of these possibilities is considered in more detail below.

Tumor angiogenesis is regulated by a number of proangiogenic factors in addition to VEGF, including fibroblast growth factors (FGFs), hepatocyte growth factor (HGF), angiopoietins (Ang), interleukin 8 (IL-8), and epidermal growth factor (EGF) $(9,10)$. Almost all human tumor cells produce VEGF, and blockade of VEGF signaling alone has potent antitumor activity against xenografted tumors in rodent models. Nevertheless, this strategy has little effect on human tumors, leading to the concept of evolution of proangiogenic factor dependence following blockade of a single family of growth factors $(11,12)$. For example, pancreatic islet tumors which developed resistance to antiangiogenic VEGF blockade were sensitive to inhibition of FGF-2 signaling (13). This result clearly indicates a switch from VEGF-dependent angiogenesis to FGF-2-dependent angiogenesis in response to chronic blockade of VEGF signaling. Thus, intracellular signaling molecules acting downstream of several proangiogenic factor 


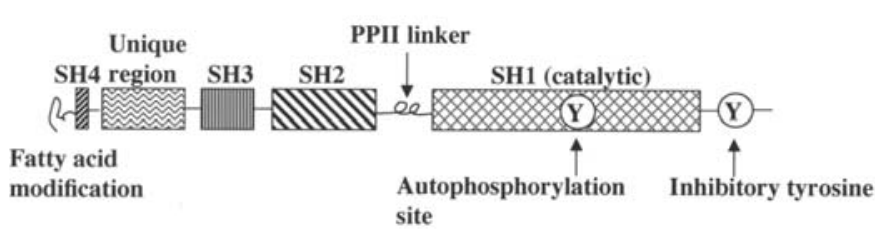

Figure 1. Structure of SFKs. Fatty acid modification of the amino terminus allows SFKs to localize to the plasma membrane. The SH3 domain binds to the PPII helix formed by the linker connecting the $\mathrm{SH} 2$ and kinase domains, while the $\mathrm{SH} 2$ domain binds to the tyrosine-phosphorylated tail. The $\mathrm{SH} 1$ domain defines the protein-tyrosine kinase domain, which is activated by autophosphorylation.

receptors may represent better targets for antiangiogenic therapy.

The second possible mechanism is that human tumors may be resistant to hypoxia, a property not modeled by tumor xenografts in rodents. This idea is supported by comparisons of oxygen content of blood in normal tissue vs. tumor. While the oxygen tension of a normal capillary bed is around $45-50 \mathrm{~mm} \mathrm{Hg}$, that of tumor endothelial cells is $5-10 \mathrm{~mm} \mathrm{Hg}$ (14). Thus endothelial cells present in human tumors may be selected for their ability to grow under hypoxic conditions. Antiangiogenic activity is usually screened by measuring the proliferation or migration of endothelial cells cultured under normoxic condition [21\% oxygen in air; $160 \mathrm{~mm} \mathrm{Hg}$; (15)]. However, chronically hypoxic endothelial cells, such as tumor endothelial cells, may be naturally resistant to certain classes of antiangiogenic molecules. Evidence for this idea is provided by examination of superficial bladder cancer. While papillary type superficial bladder cancer cells express considerable amounts of VEGF-A, the tumor is poorly vascularized. Nevertheless, few tumor cells entered apoptotic cell death despite the apparent lack of a blood supply (12). This observation suggests that while the expression of VEGF-A by bladder cancer cells may represent a hypoxic response, the tumor cells may not require VEGF-A-induced neovascularization due to intrinsic resistance to hypoxia. Such tumors are predicted to be naturally resistant to antiangiogenic therapy.

A third explanation of why antiangiogenic monotherapy does not effectively regress tumors in clinical trials may relate to the differential sensitivity of normal and tumor endothelial cells to antiangiogenic molecules. Although normal and tumorderived endothelial cells have distinct properties (16-19), whether or not the sensitivity of tumor endothelial cells to antiangiogenic molecules is lower than that of normal endothelial cells has not been established.

The above discussion suggests that many mechanisms may explain the poor efficacy of antiangiogenic therapy in human cancers. In this review, we focus on non-receptor protein-tyrosine kinases, which serve as downstream effectors for multiple proangiogenic factor receptors, and their potential as targets for future antiangiogenic drug discovery.

\section{Roles of Src family kinases in angiogenesis}

Structure of Src family kinases (SFKs). c-Src is the prototype of a non-receptor protein-tyrosine kinase family that also includes Fyn, Yes, Lyn, Lck, Hck, Blk, Fgr, and Yrk. These kinases share a conserved domain organization (Fig. 1) (20-22). At the $\mathrm{N}$-terminus there is a Src homology ( $\mathrm{SH}) 4$ domain, which is the site of myristoylation and in some cases palmitoylation, two lipid modifications essential for proper membrane targeting. SH4 is followed by $\mathrm{SH} 3, \mathrm{SH} 2$, and $\mathrm{SH} 1$ (kinase) domains and a C-terminal tail with a conserved tyrosine phosphorylation site. The SH3 domain is composed of about 60 amino acids and recognizes polyproline type II (PPII) helical motifs in target proteins. Between the SH4 and the SH3 domains, a unique region exists, which is divergent among family members and may confer specific functions. The SH2 domain, consisting of about 100 amino acids, recognizes tyrosinephosphorylated target proteins in the context of short, specific amino acid sequences. The SH1 domain defines the catalytic region, which binds ATP and substrate peptides and mediates the phosphotransfer reaction. Src family kinase activity is negatively regulated by phosphorylation of a conserved tyrosine residue in the $\mathrm{C}$-terminal tail as described in more detail below.

Regulation of kinase activity. Activation of SFKs usually occurs at the plasma membrane (20). In the inactive state, the inhibitory tyrosine in the C-terminal tail is phosphorylated by C-terminal Src kinase (CSK), inducing intramolecular binding to the SH2 domain. The SH3 domain binds to the linker connecting the SH2 and kinase domains; X-ray crystallography has shown that this linker sequence adopts the required PPII helical conformation in the context of the full-length kinase (22). Together, these two intramolecular interactions hold the kinase in a closed, inactive conformation and disruption of either is sufficient to induce kinase activation $(20,21)$. For example, dephosphorylation of the negative regulatory tail by protein-tyrosine phosphatases (PTPs), such as PTP $\alpha$ and PTP1B, causes tail release from the $\mathrm{SH} 2$ domain and kinase activation (20). SH2:tail release is also promoted by the interaction of the $\mathrm{SH} 2$ domain with other tyrosinephosphorylated proteins, such as focal adhesion kinase (FAK) (22). Thus, the presence of the phosphorylated inhibitory tyrosine, which can be detected by a phosphorylation sitespecific antibody, does not always correlate with an inactive state of SFKs. In addition, displacement of the linker PPII helix from the $\mathrm{SH} 3$ domain by other $\mathrm{SH}$-binding proteins such as HIV-1 Nef or p130 Cas (23-25), is also sufficient to induce activation of the kinase domain (Fig. 2). SH3-based activation of Src kinases does not require dephosphorylation of the C-terminal tail tyrosine residue or its release from the $\mathrm{SH} 2$ domain (26). These observations have led to the idea that SFKs act as molecular gates or switches, in which several distinct inputs (through $\mathrm{SH} 2$ or SH3) are sufficient to cause a single functional output (kinase activation).

Role of SFKs in cellular behavior of endothelial cells. Gene targeting of individual or multiple Src family members has not been reported to cause a vascular defect during embryonic development. However, SFKs may be important for adult angiogenesis, because downregulation of SFK activity by pharmacological and molecular inhibitors suppresses neovascularization in vivo (27-29). SFKs may regulate the cellular responses of endothelial cells through a number of mechanisms as described in more detail below. 


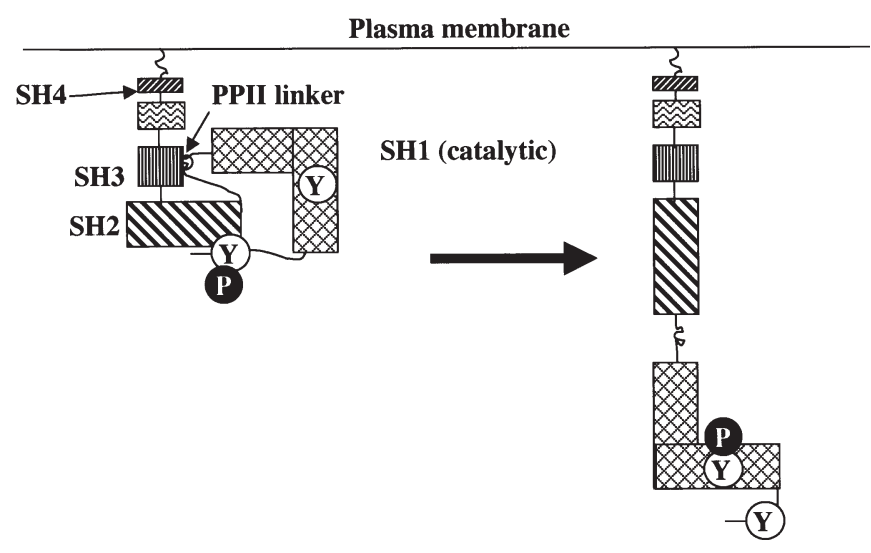

Figure 2. Conformational switching in SFKs. In the inactive state (left), the phosphorylated inhibitory tail binds to the $\mathrm{SH} 2$ domain while the PPII linker associates with the SH3 domain. Release of these intramolecular interactions can result from dephosphorylation of the tail, displacement of the tail by other tyrosine-phosphorylated proteins, or binding of proteins containing proline-rich motifs to the SH3 domain. The result is conversion of the kinase to an open, active conformation (right) that allows autophosphorylation of a conserved tyrosine residue in the activation loop of the kinase domain.

Turnover of focal adhesions (FA; assembly vs. disassembly) is an important determinant of endothelial cell behavior. For example, FA turnover influences whether cells migrate or form capillary-like structures in the murine brain capillary endothelial cell line, IBE (30). Disassembly of FA is required for proper migratory responses, whereas stabilization of FA is important for morphological differentiation (capillary morphogenesis and tube formation; Fig. 3). FGF-2 activates c-Src within FA through the c-Fes protein-tyrosine kinase (see below), leading to extracellular signal-regulated kinase (ERK) activation, FA disassembly, and chemotaxis toward FGF-2 by IBE cells $(31,32)$. Fyn is also activated by FGF-2 in a manner dependent on culture conditions (33). Active Fyn phosphorylates $\beta$-catenin, which in turn induces binding to T-cell factor-4 (TCF-4). Fibronectin is upregulated through the transcriptional activity of TCF-4; antibody-mediated inhibition of the fibronectin receptor ( $\alpha 5 \beta 1$ integrin) attenuates
FGF-2-induced tube formation by IBE cells (34). Together, these results implicate Fyn in the FGF-2 pathway leading to capillary formation. Similarly, inhibition of $\alpha 5 \beta 1$ integrin resulted in disorganized microvessel formation in FGF-2containing Matrigel plugs in mice (34). Thus, FGF-2-activated SFKs are likely to be involved in migration and differentiation by endothelial cells.

VEGF-A signals through the VEGF receptor 2 [VEGFR2; also designated as kinase insert-domain-containing receptor (KDR) in humans or fetal liver kinase 1 (Flk-1) in mice]. Expression of VEGFR2 at the cell surface is a marker for activated endothelial cells in vivo, and is linked to SFK activation in a number of different biological contexts. VEGF-A stimulates the trafficking of VEGFR2 from endosomal storage to the cell surface through activated Src in human umbilical vein endothelial cells (HUVECs) (35). Activated VEGFR2 recruits Fyn and activates p38 MAPK, leading to endothelial cell migration (36). VEGF-A also activates c-Src by recruitment to VEGFR2, leading to nitric oxide production in bovine aortic endothelial cells (37). VEGF-A-induced Src activation contributes to FAK activation, which in turn regulates the motility and survival of HUVECs (38). VEGF-A also activates diacylglycerol kinase $\alpha$ through $\mathrm{Src}$ in porcine aortic endothelial (PAE) cells and HUVECs, leading to migration and capillary morphogenesis (39). Down-regulation of c-Yes by small interfering RNA (siRNA) attenuates VEGF-Amediated migration, whereas down-regulation of Fyn increases migration and inhibits tube formation by human retinal microvascular endothelial cells (40), suggesting nonredundant and possibly opposing functions for individual $\mathrm{Src}$ family members in this cell type. In summary, VEGF-Ainduced SFK activation via VEGFR2 controls endothelial cell migration, survival, nitric oxide production, and differentiation.

Hepatocyte growth factor (HGF) is a potent proangiogenic factor, which activates the HGF receptor tyrosine kinase encoded by the protooncogene, $c$-met. HGF promotes nitric oxide production through $\mathrm{Src}$, which regulates HUVEC proliferation (41). HGF activates the c-Jun animo-terminal kinase (JNK) through Src, leading to migration of human brain microvascular endothelial cells (42). Expression of
A. Tube formation

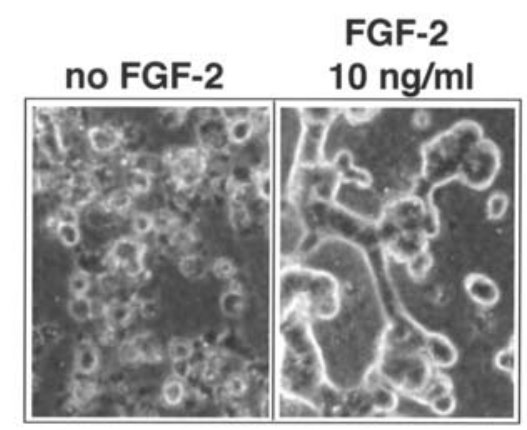

Collagen gels

\section{B. Capillary morphogenesis}

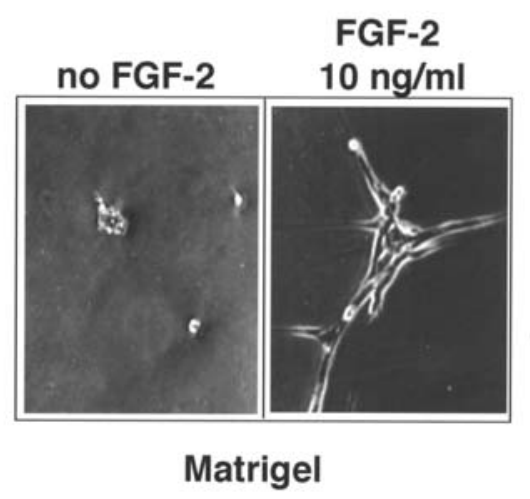

Figure 3. Morphological differentiation of IBE cells. (A) IBE cells were cultured between two layers of type I collagen gels for $18 \mathrm{~h}$ in the presence or absence of FGF-2. FGF-2-treated IBE cells form cell aggregates, followed by the formation of tight cell-cell contacts (compaction). The aggregates then fuse to each other to form cord-like structures, followed by lumen formation (71). (B) IBE cells were cultured on the surface of growth factor-reduced Matrigel for $24 \mathrm{~h}$ in the presence or absence of FGF-2. FGF-2-treated IBE cells extend their morphology, and neighboring cells fuse and form a slit between them. FGF-2dependent stabilization of FA is important to maintain this extended morphology (30). 


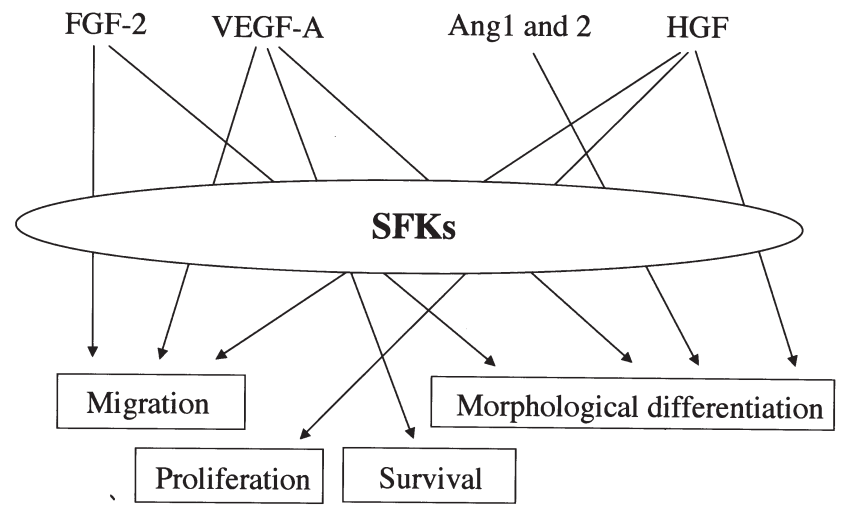

Figure 4. FGF-2, VEGF-A, Ang1 and 2, and HGF promote diverse biological responses of endothelial cells through SFKs.

kinase-inactive Src, treatment with the Src inhibitor PP2, and downregulation of Src by siRNA all inhibited HGF-induced capillary morphogenesis by the murine spleen endothelial cell line, MSS31 and by HUVECs (43). Thus, it is likely that Src regulates HGF-induced proliferation, migration, and differentiation by endothelial cells.

Angiopoietins (Ang) belong to a family of ligands for the receptor tyrosine kinase Tie 2. Ang1 and Ang2 are expressed in endothelial cells within tumor tissues, suggesting that they may have roles in tumor angiogenesis. The functional significance of Src family kinases in Ang-driven angiogenesis has not been fully elucidated. Recently, we found that Ang1activated Src was responsible for capillary morphogenesis by HUVECs (44). Using IBE cells, we found that Fyn may be required for Ang2-induced tube formation (45).

Aside from ligands for receptor tyrosine kinases, other classes of proangiogenic factors also utilize SFKs as signaling molecules. Norepinephrine indirectly stimulates angiogenesis by expression of the VEGF-A gene through cAMP-dependent activation of Src in mouse brown adipocytes (46). Soluble E-selectin (sE-selectin) mediates angiogenesis by stimulating chemotaxis and tube formation by human dermal microvascular endothelial cells (HDMVECs). Both of these responses are blocked by the Src inhibitor PP2 and by Src antisense oligonucleotides (47). Interstitial collagen I stimulates capillary morphogenesis by HDMVECs through Src (48). Heparin affin regulatory peptide is an $18-\mathrm{kDa}$ growth factor for which protein-tyrosine phosphatase beta/zeta serves as receptor. This phosphatase activates Src, most likely through tail dephosphorylation as described above, which in turn regulates migration and tube formation of HUVECs (49). The cytochrome P450 2C9 metabolite of arachidonic acid, 14,15epoxyeicosatrienoic acid, induces migration and tube formation of HDMVECs by Src-dependent production of FGF-2 (50). Endothelin-1 stimulates migration of human brain-derived microvascular endothelial cells, which requires Src (51). Thus, SFKs are common signaling molecules for a wide variety of proangiogenic factors. Blockade of Src family kinases may efficiently inhibit tumor angiogenesis driven by several proangiogenic factor receptors, such as VEGFR2, FGF receptor, c-Met, and Tie 2 (Fig. 4) as well as other mediators.
Inhibition of SFK in anticancer therapy. SFKs play pivotal roles in angiogenesis as well as in tumor progression. Specifically, SFKs are implicated in migration, survival, and upregulation of matrix metalloproteases by tumor cells, leading to invasion and metastasis (52-54). SFKs are activated and their expression is elevated in a variety of human cancers (55). In preclinical animal models, inhibition of SFKs reduced tumor progression and metastasis along with angiogenesis (56-59), indicating that inhibition of SFKs is a promising strategy for anti-cancer therapy.

Several Src inhibitors are now entering clinical trials $(60,61)$. These compounds are usually designed to compete for ATP binding in the kinase domain. Because of the structural similarity of kinase domains, small molecules targeting the ATP-binding pocket may produce off-target effects on other kinases. While this lack of specificity may contribute to toxicity, it may also contribute to anti-tumor efficacy by blocking related members of the same kinase family, thus suppressing redundant pathways such as those controlled by Src, Fyn and Yes downstream of proangiogenic factors as described above. Practical advantages of this class of inhibitors may include oral administration and low cost in comparison to protein-based therapies such as monoclonal antibodies.

Inhibitors targeting other protein-tyrosine kinase and serine/ threonine kinase targets have been investigated as possible antiangiogenic therapies $(62,63)$. Although they showed potent antiangiogenic activities and subsequent tumor regression in preclinical models, many of them failed to regress pre-existing tumors or improve survival in human clinical trials. One reason for this discrepancy may relate to the altered inhibitor sensitivity of human tumor endothelial cells exposed to long-term hypoxia. We tested this possibility using endothelial cells cultured under either normoxic $\left(21 \% \mathrm{O}_{2}, 160 \mathrm{~mm} \mathrm{Hg}\right)$ or hypoxic $\left(1.5 \% \mathrm{O}_{2}\right.$, $11 \mathrm{~mm} \mathrm{Hg}$ ) conditions $(14,15)$. Although the down-regulation of Src by siRNA in HUVECs grown under both normoxic and hypoxic conditions reduced Ang1-induced capillary morphogenesis, the Src inhibitor PP2 failed to inhibit capillary morphogenesis by HUVECs cultured under hypoxic condition. In these cells, the multidrug-resistance associated protein 1 (MRP-1) was upregulated and treatment with verapamil or siRNA for MRP-1 restored the inhibitory action of PP2 on Ang1-induced capillary morphogenesis by HUVECs cultured under hypoxic conditions (44). These results suggest that hypoxia-induced upregulation of $\mathrm{ABC}$ transporters may contribute to resistance to small molecule protein kinase inhibitors in human tumor endothelial cells. Combining inhibitors of $\mathrm{ABC}$ transporters and tyrosine kinases may therefore increase efficacy in clinical trials.

\section{Roles of Fes in angiogenesis}

Structure and regulation of the c-Fes tyrosine kinase. Fes (also known as Fps) and Fer define a unique subfamily of non-receptor protein-tyrosine kinases $(64,65)$. While Fer is expressed ubiquitously, Fes expression is restricted to myeloid hematopoietic cells, vascular endothelial cells, some neuronal cell types and epithelial cells (66). Structurally, Fes consists of a long N-terminal unique region followed by an $\mathrm{SH} 2$ domain and a protein-tyrosine kinase domain with two autophosphorylation sites [Y713 and Y811; Fig. 5; $(64,65)$ ]. Mutation 


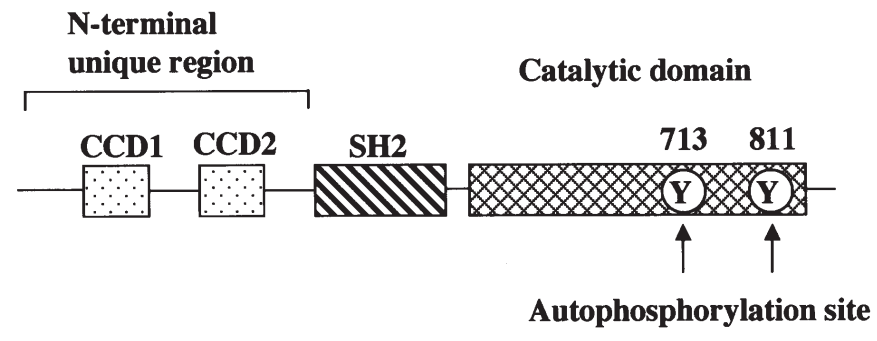

Figure 5. Structure of the c-Fes tyrosine kinase. The unique amino-terminal region of Fes contains two coiled-coil domains (CCD). These oligomerization domains may be involved in intermolecular or intramolecular Fes association and contribute to kinase regulation and substrate recruitment. The $\mathrm{SH} 2$ domain is located between the protein-tyrosine kinase domain and the amino-terminal unique region. Two major autophosphorylation sites are present in the kinase domain; Y713 is found in a consensus activation loop sequence and is critical for kinase activity and biological function.

of Y713 reduced both autophosphorylation of Y811 and phosphorylation of a Fes substrate, suggesting that Y713 is a key regulatory residue for Fes kinase activity. Wild-type Fes transphosphorylated both Y713 and Y811 of kinaseinactive Fes (67) and has been shown to undergo homo-oligomerization via its $\mathrm{N}$-terminal unique region (68), indicating that Fes is activated by autophosphorylation through an intermolecular mechanism. Oligomerization of Fes is mediated by coiled-coil homology domains (CCDs) found in the unique $\mathrm{N}$-terminal region (Fig. 5). A point mutation in the first CCD which disrupts coiled-coil formation (L145P) markedly increased Fes tyrosine kinase activity, whereas a similar point mutation in the second CCD (L344P) had little effect on its own (69). However, a combination of both mutations (2LP) reversed the activating effect of the L145P mutation, suggesting that the first CCD negatively regulates kinase activity while the second $\mathrm{CCD}$ has a positive regulatory role. The kinase activities of these mutants paralleled their biological activities in a fibroblast focus-forming assay and their ability to drive granulocyte-macrophage colony-stimulating factor-independent survival of TF-1 myeloid leukemia cells (69). Regulation of protein-tyrosine kinase activity by coiled-coil domains is a unique defining feature of the Fes/Fer kinase family.

Regulation of Fes kinase activity in FGF-2-treated endothelial cells. In living cells, the basal tyrosine kinase activity of Fes is tightly repressed. We observed that IBE brain capillary endothelial cells express c-Fes and that FGF-2 induced autophosphorylation of Fes when cultured on fibronectin (FN)coated plastic (70). In contrast, FGF-2 failed to activate either endogenous or transfected Fes in IBE cells stably expressing a kinase-inactive Fes mutant (KD-Fes cells), suggesting that the kinase-dead mutant exerted a dominant negative effect on endogenous Fes (70).

IBE cells express the FGF receptor 1 (FGFR1) and IIIc type FGFR2 (71), which are both activated by FGF-2. IBE cells do not express platelet-derived growth factor (PDGF) receptor- $\alpha$ or IIIb type FGFR2, to which FGF-1, FGF-7 (keratinocyte growth factor), and FGF-10, but not FGF-2, exclusively bind (72). To examine the signal transduction pathways regulated through either FGFR1 or R2 alone, we established stable cell lines expressing either PDGF receptor- $\alpha /$ FGFR1 chimera [extracellular domain of PDGF receptor- $\alpha$ was fused to intra-

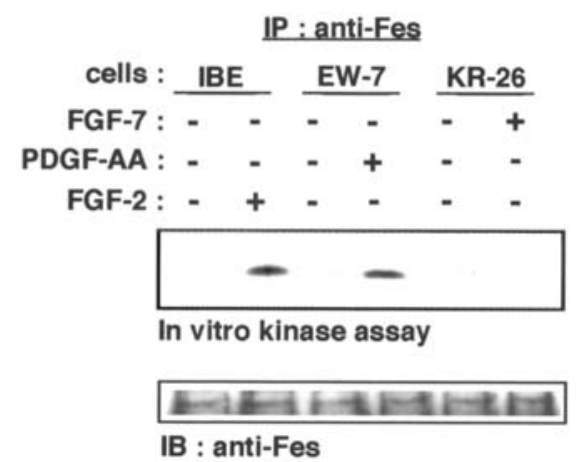

Figure 6. FGFR1 is responsible for the activation of Fes in endothelial cells. Both FGFR1 and 2 are expressed in endothelial cells. To examine the unique signals downstream of these receptor tyrosine kinases, we stably expressed either PDGF receptor- $\alpha /$ FGFR1 chimera (extracellular domain of PDGF receptor- $\alpha$ was fused to intracellular domain of FGFR1; clone EW-7) or the IIIb FGFR2 receptor (clone KR-26) in IBE cells. These cells were cultured on the surface of fibronectin-coated dishes, stimulated with either PDGF-AA or FGF-7, and autophosphorylation of endogenous Fes was examined by immune complex kinase assay.

cellular domain of FGFR1; (71)] or IIIb FGFR2 (73). These cells were stimulated with either PDGF-AA or FGF-7 and autophosphorylation of endogenous Fes was examined by immune complex kinase assay. As shown in Fig. 6, PDGF-AA but not FGF-7 activated Fes, indicating that the intracellular domain of FGFR1 was responsible for FGF-2-mediated activation of Fes in endothelial cells. We also made stable cell lines expressing the Fes coiled-coil domain mutants L145P, L344P, or 2LP described above. As shown in Fig. 7, the basal kinase activities of these Fes mutants were comparable with those observed following expression in TF-1 myeloid leukemia cells, suggesting that Fes regulation by coiled-coils is independent of cell lineage. FGF-2 treatment induced autophosphorylation of wild-type Fes but did not further activate the mutant variants of Fes. One interpretation of these results is that coupling of Fes to the FGF-2 receptor and liganddependent regulation may require intact coiled-coil domains.

Role of Fes in cellular responses of endothelial cells. Targeted deletion of the Fes gene did not cause abnormal development of cardiovascular system $(74,75)$, suggesting that Fes is not required for vascular development per se. However, expression of an artificially membrane-targeted form of Fes in transgenic mice increased vascularity and led to hemangioma formation (76), suggesting that Fes may be involved in angiogenesis in vivo. We examined the effect of proangiogenic factors on angiogenic cellular responses of endothelial cells using IBE cells expressing dominant-negative Fes as described above (KD-Fes cells). FGF-2-induced chemotaxis was impaired in $\mathrm{KD}-\mathrm{Fes}$ cells (70). However, when KD-Fes cells were cultured in collagen gels, differentiation to tube-like structures was still observed in response to FGF-2-treatment. In addition, overexpression of wild-type Fes led to tube formation by IBE cells in the absence of FGF-2 (70). These results suggest that Fes activity may contribute to endothelial cell migration and differentiation in vitro.

Ang2 activated PI3-kinase in IBE cells expressing wild-type Fes but not KD-Fes (45). Activated PI3-kinase associated with wild-type Fes, but not with Tie 2 (45), which was previously 


\section{IP: anti-FLAG (M2)}

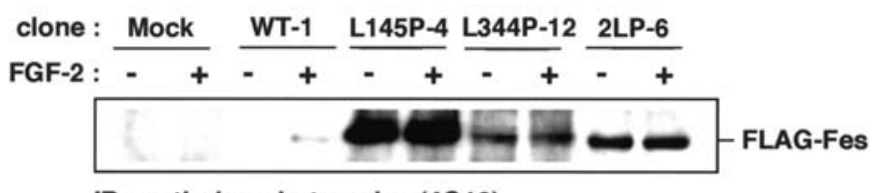

IB : anti-phosphotyrosine (4G10)

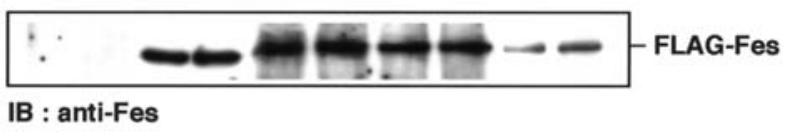

Figure 7. Mutations in the CCD disrupt negative regulation of Fes kinase activity in IBE cells. IBE cells stably expressing wild-type (WT) Fes, a first CCD mutant (L145P), a second CCD mutant (L344P), or the double CCD mutant (2LP) were cultured on the surface of fibronectin-coated dishes, stimulated with FGF-2, and autophosphorylation of endogenous Fes was examined by immunoblotting. Note that while wild-type Fes can be regulated by FGF-2 in these cells, all of the CCD mutants show enhanced autophosphorylation in the absence of FGF-2 treatment. This result suggests that the CCDs are essential for proper downregulation of Fes kinase activity and coupling to FGFRs

identified as a PI3-kinase binding protein in Ang1-treated cells $(77,78)$. Ang2-activated PI3-kinase was involved in chemotaxis toward Ang2. We also examined the effect of Ang1 on the association of Tie 2 with PI3-kinase. Ang1 increased PI3-kinase activity and induced co-immunoprecipitation with endogenous Fes but not with Tie 2 (79). Thus, PI3-kinase activation in response to Ang treatment leads primarily to association with Fes in endothelial cells. Blockade of Angdependent PI3K activation by KD-Fes suggests a necessary role for Fes kinase activity in this pathway (Fig. 8).

VEGF-A induced autophosphorylation of Fes through VEGFR2 but not VEGFR1 in porcine aortic endothelial cells (PAE cells) (80). As described above for Ang2, VEGF-Ainduced PI3-kinase activation was partly mediated through wild-type Fes. Other molecules, such as VEGFR2, Src, and insulin receptor substrate-1, were also found to associate with PI3-kinase. These molecules may be involved in VEGF-A- induced PI3-kinase activation, since expression of kinaseinactive Fes showed no dominant negative effect on VEGF-Ainduced PI3-kinase activation, chemotaxis toward VEGF-A, or VEGF-A-mediated capillary morphogenesis (80). Interestingly, expression of wild-type Fes in PAE cells promoted VEGF-A-independent capillary morphogenesis (80), similar to FGF-2-independent tube formation of IBE cells (70).

Stromal cell-derived factor- $1 \alpha(\mathrm{SDF}-1 \alpha)$ is a CXC chemokine that promotes angiogenesis and also vasculogenesis by circulating endothelial progenitor cells $(81,82)$. Targeted disruption of a gene encoding the receptor for SDF-1 $\alpha$, CXCR4, causes impaired intestinal vascular development (83). CXCR4 is a G-protein coupled receptor and is expressed in endothelial cells. We observed that treatment of endothelial cells with SDF-1 $\alpha$ increased PI3-kinase activity along with tube formation by IBE cells and capillary morphogenesis by HUVECs (84). Inhibition of PI3-kinase attenuated SDF-1 $\alpha$-mediated tube formation and capillary morphogenesis. Importantly, SDF-1 $\alpha-$ induced PI3-kinase activation was dependent on the Fes activity, since SDF- $1 \alpha$ failed to activate PI3-kinase and to induce tube formation in KD-Fes cells (84). SDF-1 $\alpha$ also promoted tight cell-cell contact (compaction), which is involved in tube formation.

Hedgehog family cytokines, consisting of Sonic hedgehog (Shh), Indian hedgehog, and desert hedgehog, regulate the morphology of a variety of cell types and may contribute indirectly to angiogenesis (85). However, hedgehog signaling is required for embryonic vascular development (86-88), suggesting that it may directly regulate the morphology of endothelial cells. We found that Shh-induced tube formation by cultured endothelial cells correlates with PI3-kinase activation in a Fes-dependent manner (unpublished data). We also observed that Shh induced capillary morphogenesis via the Fes/PI3-kinase pathway (89). Thus, Fes acts as a common signaling molecule downstream of several proangiogenic factor receptors.

Fes in antiangiogenic strategy. Based on the results described above, inhibition of Fes activity in angiogenic endothelial

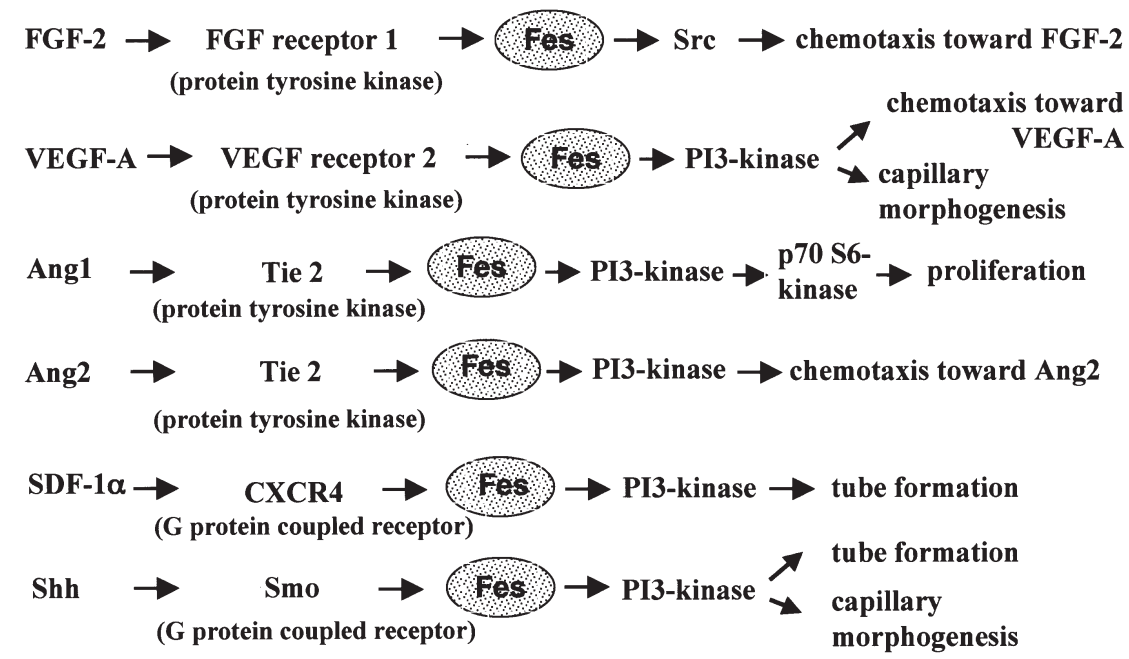

Figure 8. Fes is a common signaling molecule downstream of several proangiogenic factor receptors expressed in endothelial cells. Fes is involved in the activation of PI3-kinase by several proangiogenic factors in endothelial cells with the exception of FGF-2. In this case, Fes contributes to the activation of Src within FA. 
cells may represent an attractive strategy for antiangiogenic therapy. Tumor-infiltrating macrophages induce angiogenesis $(90,91)$, and cytokines that stimulate proliferation, migration, and activation of macrophages also utilize Fes as a common signal transducer (92). Therefore, inhibition of Fes activity may inhibit macrophage-driven angiogenesis in addition to direct effects on angiogenic signaling pathways in endothelial cells. Recent findings suggest that circulating endothelial precursor cells mobilized from bone marrow significantly contribute to the new microvessel formation in tumor tissues (93). VEGF-A and SDF-1 $\alpha$ play pivotal roles in this process (94). Because these cells are also likely to express Fes, inhibition of its kinase activity may block endothelial precursor cell-dependent vasculogenesis in tumors.

Unlike SFKs, small molecule protein kinase inhibitors that selectively inhibit Fes have yet to be identified. Previously work has shown that the non-selective protein-tyrosine kinase inhibitor herbimycin A inhibited fibroblast transformation by v-Fps, the transforming avian retroviral homolog of c-Fes (95). We examined the effects of a panel of tyrosine kinase inhibitors including herbimycin A, PP2, and SU6656, on autophosphorylation of L145P Fes expressed in PAE cells. However, none of them inhibited its autophosphorylation at non-toxic concentrations (S. Kanda, unpublished data). While these inhibitors are active against Src family kinases (and Abl in the case of PP2), their inability to block Fes kinase activity suggests novel structural subtleties in the ATP binding pocket. Evidence for such a conclusion requires the structure of the Fes kinase domain.

To establish an antiangiogenic strategy for the treatment of cancer patients, the net effect of the inhibition of Fes activity on the proliferation of tumor cells should be examined, because certain antiangiogenic therapies may enhance tumor progression in vivo. Recently, somatic mutations of Fes were found in human colorectal cancers (96). Although fes was originally identified as an avian and feline retroviral oncogene, the colorectal cancer-associated mutations paradoxically reduced Fes kinase activity $(97,98)$. Furthermore, introduction of kinase-inactivating Fes mutations in a mouse model of breast cancer reduced the latency for tumor formation, an effect that was rescued by a wild-type fes transgene (97). Restoring wild-type Fes expression to colorectal cancer cell lines inhibited anchorage-independent growth in soft agar (98) and invasion through Matrigel (unpublished data). However, whether or not Fes kinase activity is required for these apparent tumor-suppressor functions is currently unclear. Nevertheless, these observations suggest that inhibition of Fes kinase activity in vivo may promote epithelial tumor growth and progression, even though inhibition of this activity in vascular endothelial cells may produce a therapeutic benefit in terms of an antiangiogenic effect as outlined above. Discovery of Fes-selective kinase inhibitors is an essential first step towards understanding the net biological effect of simultaneous blockade of Fes-mediated angiogenic and tumor-suppressor functions in vivo.

\section{Acknowledgements}

This work was supported by Grants-in-Aid for Scientific Research from the Japan Society of the Promotion of Science (to S.K. and Y.M.) and by grants from the National Institutes of Health (T.E.S.)

\section{References}

1. Folkman $\mathrm{J}$ : What is the evidence that tumors are angiogenesis dependent? J Natl Cancer Inst 82: 4-6, 1990.

2. Bikfalvi A: Significance of angiogenesis in tumour progression and metastasis. Eur J Cancer 31A: 1101-1104, 1995.

3. Carmeliet P: Angiogenesis in health and disease. Nat Med 9: 653-660, 2003.

4. Bicknell R and Harris AL: Mechanisms and therapeutic implications of angiogenesis. Curr Opin Oncol 8: 60-65, 1996.

5. Folkman J: Seminars in Medicine of the Beth Israel Hospital, Boston. Clinical applications of research on angiogenesis. N Engl J Med 333: 1757-1763, 1995.

6. Ferrara N and Davis-Smyth T: The biology of vascular endothelial growth factor. Endocr Rev 18: 4-25, 1997.

7. Yang JC, Haworth L, Sherry RM, Hwu P, Schwartzentruber DJ, Topalian SL, Steinberg SM, Chen HX and Rosenberg SA: A randomized trial of bevacizumab, an anti-vascular endothelial growth factor antibody, for metastatic renal cancer. N Engl J Med 349: 427-434, 2003.

8. Cobleigh MA, Langmuir VK, Sledge GW, Miller KD, Haney L, Novotny WF, Reimann JD and Vassel A: A phase I/II doseescalation trial of bevacizumab in previously treated metastatic breast cancer. Semin Oncol 30: 117-124, 2003.

9. Colville-Nash PR and Willoughby DA: Growth factors in angiogenesis: current interest and therapeutic potential. Mol Med Today 3: 14-23, 1997.

10. Ferrara N and Alitalo K: Clinical applications of angiogenic growth factors and their inhibitors. Nat Med 5: 1359-1364, 1999.

11. Cao Y: Antiangiogenic cancer therapy. Semin Cancer Biol 14: 139-145, 2004.

12. Kanda S, Miyata Y and Kanetake H: Studies of the endothelial cell-specific signal transduction pathways. New paradigm for the development of potent anti-angiogenic therapies. In: Trends in Angiogenesis Research. Zubar RV (ed.) Nova Science Publishers, Inc., New York, pp43-69, 2005.

13. Casanovas O, Hicklin DJ, Bergers G and Hanahan D: Drug resistance by evasion of antiangiogenic targeting of VEGF signaling in late-stage pancreatic islet tumors. Cancer Cell 8: 299-309, 2005.

14. Hockel M and Vaupel P: Tumor hypoxia: definitions and current clinical, biologic, and molecular aspects. J Natl Cancer Inst 93: 266-276, 2001.

15. Blagosklonny MV: Antiangiogenic therapy and tumor progression. Cancer Cell 5: 13-17, 2004.

16. St Croix B, Rago C, Velculescu V, Traverso G, Romans KE, Montgomery E, Lal A, Riggins GJ, Lengauer C, Vogelstein B and Kinzler KW: Genes expressed in human tumor endothelium. Science 289: 1197-1202, 2000.

17. Carson-Walter EB, Watkins DN, Nanda A, Vogelstein B, Kinzler KW and St Croix B: Cell surface tumor endothelial markers are conserved in mice and humans. Cancer Res 61: 6649-6655, 2001.

18. Oh P, Li Y, Yu J, Durr E, Krasinska KM, Carver LA, Testa JE and Schnitzer JE: Subtractive proteomic mapping of the endothelial surface in lung and solid tumours for tissue-specific therapy. Nature 429: 629-635, 2004.

19. Hida K, Hida Y, Amin DN, Flint AF, Panigrahy D, Morton CC and Klagsbrun M: Tumor-associated endothelial cells with cytogenetic abnormalities. Cancer Res 64: 8249-8255, 2004.

20. Bjorge JD, Jakymiw A and Fujita DJ: Selected glimpses into the activation and function of Src kinase. Oncogene 19: 5620-5635, 2000.

21. Boggon TJ and Eck MJ: Structure and regulation of Src family kinases. Oncogene 23: 7918-7927, 2004.

22. Chong YP, Ia KK, Mulhern TD and Cheng HC: Endogenous and synthetic inhibitors of the Src-family protein tyrosine kinases. Biochim Biophys Acta 1754: 210-220, 2005.

23. Briggs SD, Sharkey M, Stevenson M and Smithgall TE: SH3mediated Hck tyrosine kinase activation and fibroblast transformation by the Nef protein of HIV-1. J Biol Chem 272: 17899-17902, 1997.

24. Moarefi I, La Fevre-Bernt M, Sicheri F, Huse M, Lee CH, Kuriyan J and Miller WT: Activation of the Src-family tyrosine kinase Hck by SH3 domain displacement. Nature 385: 650-653, 1997. 
25. Pellicena P and Miller WT: Processive phosphorylation of p130Cas by Src depends on SH3-polyproline interactions. J Biol Chem 276: 28190-28196, 2001

26. Lerner EC and Smithgall TE: SH3-dependent stimulation of Src-family kinase autophosphorylation without tail release from the SH2 domain in vivo. Nat Struct Biol 9: 365-369, 2002.

27. Eliceiri BP, Paul R, Schwartzberg PL, Hood JD, Leng J and Cheresh DA: Selective requirement for Src kinases during VEGFinduced angiogenesis and vascular permeability. Mol Cell 4: 915-924, 1999.

28. Kilarski WW, Jura N and Gerwins P: Inactivation of Src family kinases inhibits angiogenesis in vivo: implications for a mechanism involving organization of the actin cytoskeleton. Exp Cell Res 291: 70-82, 2003.

29. Werdich XQ and Penn JS: Specific involvement of SRC family kinase activation in the pathogenesis of retinal neovascularization. Invest Ophthalmol Vis Sci 47: 5047-5056, 2006.

30. Kanda S, Miyata Y and Kanetake H: Role of focal adhesion formation in migration and morphogenesis of endothelial cells. Cell Signal 16: 1273-1281, 2004.

31. Shono T, Kanetake H and Kanda S: The role of mitogen-activated protein kinase activation within focal adhesions in chemotaxis toward FGF-2 by murine brain capillary endothelial cells. Exp Cell Res 264: 275-283, 2001.

32. Kanda S, Miyata Y, Kanetake H and Smithgall TE: Fibroblast growth factor-2 induces the activation of Src through Fes, which regulates focal adhesion disassembly. Exp Cell Res 312: 3015-3022, 2006.

33. Tsuda S, Ohtsuru A, Yamashita S, Kanetake H and Kanda S: Role of c-Fyn in FGF-2-mediated tube-like structure formation by murine brain capillary endothelial cells. Biochem Biophys Res Commun 290: 1354-1360, 2002.

34. Kanda S, Miyata Y and Kanetake H: T-cell factor-4-dependent up-regulation of fibronectin is involved in fibroblast growth factor-2-induced tube formation by endothelial cells. J Cell Biochem 94: 835-847, 2005.

35. Gampel A, Moss L, Jones MC, Brunton V, Norman JC and Mellor H: VEGF regulates the mobilization of VEGFR2/KDR from an intracellular endothelial storage compartment. Blood 108: 2624-2631, 2006.

36. Lamalice L, Houle F and Huot J: Phosphorylation of Tyr1214 within VEGFR-2 triggers the recruitment of Nck and activation of Fyn leading to SAPK2/p38 activation and endothelial cell migration in response to VEGF. J Biol Chem 281: 34009-34020, 2006.

37. He H, Venema VJ, Gu X, Venema RC, Marrero MB and Caldwell RB: Vascular endothelial growth factor signals endothelial cell production of nitric oxide and prostacyclin through flk1/KDR activation of c-Src. J Biol Chem 274: 25130-25135, 1999.

38. Abu-Ghazaleh R, Kabir J, Jia H, Lobo M and Zachary I: Src mediates stimulation by vascular endothelial growth factor of the phosphorylation of focal adhesion kinase at tyrosine 861, and migration and anti-apoptosis in endothelial cells. Biochem J 360: 255-264, 2001.

39. Baldanzi G, Mitola S, Cutrupi S, Filigheddu N, van Blitterswijk WJ, Sinigaglia F, Bussolino $\mathrm{F}$ and Graziani A: Activation of diacylglycerol kinase alpha is required for VEGF-induced angiogenic signaling in vitro. Oncogene 23: 4828-4838, 2004.

40. Werdich XQ and Penn JS: Src, Fyn and Yes play differential roles in VEGF-mediated endothelial cell events. Angiogenesis 8: 315-326, 2005.

41. Maejima Y, Ueba H, Kuroki M, Yasu T, Hashimoto S, Nabata A, Kobayashi N, Ikeda N, Saito M and Kawakami M: Src family kinases and nitric oxide production are required for hepatocyte growth factor-stimulated endothelial cell growth. Atherosclerosis 167: 89-95, 2003

42. Rush S, Khan G, Bamisaiye A, Bidwell P, Leaver HA and Rizzo MT: c-jun amino-terminal kinase and mitogen activated protein kinase $1 / 2$ mediate hepatocyte growth factor-induced migration of brain endothelial cells. Exp Cell Res 313: 121-132, 2007.

43. Kanda S, Kanetake H and Miyata Y: HGF-induced capillary morphogenesis of endothelial cells is regulated by Src. Biochem Biophys Res Commun 344: 617-622, 2006.

44. Kanda S, Kanetake H and Miyata Y: Role of Src in angiopoietin 1induced capillary morphogenesis of endothelial cells: effect of chronic hypoxia on Src inhibition by PP2. Cell Signal 19: 472-480, 2007.

45. Mochizuki Y, Nakamura T, Kanetake H and Kanda S: Angiopoietin 2 stimulates migration and tube-like structure formation of murine brain capillary endothelial cells through c-Fes and c-Fyn. J Cell Sci 115: 175-183, 2002.
46. Fredriksson JM, Lindquist JM, Bronnikov GE and Nedergaard J: Norepinephrine induces vascular endothelial growth factor gene expression in brown adipocytes through a beta-adrenoreceptor/cAMP/protein kinase A pathway involving Src but independently of Erk1/2. J Biol Chem 275: 13802-13811, 2000.

47. Kumar P, Amin MA, Harlow LA, Polverini PJ and Koch AE: Src and phosphatidylinositol 3-kinase mediate soluble Eselectin-induced angiogenesis. Blood 101: 3960-3968, 2003.

48. Liu Y and Senger DR: Matrix-specific activation of Src and Rho initiates capillary morphogenesis of endothelial cells. FASEB J 18: 457-468, 2004.

49. Polykratis A, Katsoris P, Courty J and Papadimitriou E: Characterization of heparin affin regulatory peptide signaling in human endothelial cells. J Biol Chem 280: 22454-22461, 2005.

50. Zhang B, Cao H and Rao GN: Fibroblast growth factor-2 is a downstream mediator of phosphatidylinositol 3-kinase-Akt signaling in 14,15-epoxyeicosatrienoic acid-induced angiogenesis. J Biol Chem 281: 905-914, 2006.

51. Milan J, Charalambous C, Elhag R, Chen TC, Li W, Guan S, Hofman FM and Zidovetzki R: Multiple signaling pathways are involved in endothelin-1-induced brain endothelial cell migration. Am J Physiol 291: C155-C164, 2006.

52. Frame MC: Newest findings on the oldest oncogene; how activated src does it. J Cell Sci 117: 989-998, 2004.

53. Playford MP and Schaller MD: The interplay between Src and integrins in normal and tumor biology. Oncogene 23: 7928-7946, 2004.

54. Summy JM and Gallick GE: Treatment for advanced tumors: SRC reclaims center stage. Clin Cancer Res 12: 1398-1401, 2006.

55. Irby RB and Yeatman TJ: Role of Src expression and activation in human cancer. Oncogene 19: 5636-5642, 2000.

56. Yezhelyev MV, Koehl G, Guba M, Brabletz T, Jauch KW, Ryan A, Barge A, Green T, Fennell M and Bruns CJ: Inhibition of SRC tyrosine kinase as treatment for human pancreatic cancer growing orthotopically in nude mice. Clin Cancer Res 10: 8028-8036, 2004

57. Summy JM, Trevino JG, Lesslie DP, Baker CH, Shakespeare WC, Wang Y, Sundaramoorthi R, Metcalf CA III, Keats JA, Sawyer TK and Gallick GE: AP23846, a novel and highly potent Src family kinase inhibitor, reduces vascular endothelial growth factor and interleukin-8 expression in human solid tumor cell lines and abrogates downstream angiogenic processes. Mol Cancer Ther 4: 1900-1911, 2005

58. Trevino JG, Summy JM, Lesslie DP, Parikh NU, Hong DS, Lee FY, Donato NJ, Abbruzzese JL, Baker CH and Gallick GE: Inhibition of SRC expression and activity inhibits tumor progression and metastasis of human pancreatic adenocarcinoma cells in an orthotopic nude mouse model. Am J Pathol 168: 962-972, 2006.

59. Han LY, Landen CN, Trevino JG, Halder J, Lin YG, Kamat AA, Kim TJ, Merritt WM, Coleman RL, Gershenson DM, Shakespeare WC, Wang Y, Sundaramoorth R, Metcalf CA III, Dalgarno DC, Sawyer TK, Gallick GE and Sood AK: Antiangiogenic and antitumor effects of SRC inhibition in ovarian carcinoma. Cancer Res 66: 8633-8639, 2006.

60. Lee D and Gautschi O: Clinical development of SRC tyrosine kinase inhibitors in lung cancer. Clin Lung Cancer 7: 381-384, 2006.

61. Alvarez RH, Kantarjian HM and Cortes JE: The role of Src in solid and hematologic malignancies: development of newgeneration Src inhibitors. Cancer 107: 1918-1929, 2006.

62. Smith JK, Mamoon NM and Duhe RJ: Emerging roles of targeted small molecule protein-tyrosine kinase inhibitors in cancer therapy. Oncol Res 14: 175-225, 2004.

63. Kanda S, Miyata Y and Kanetake: Current status and perspective of antiangiogenic therapy for cancer: urinary cancer. Int J Clin Oncol 11: 90-107, 2006.

64. Smithgall TE, Rogers JA, Peters KL, Li J, Briggs SD, Lionberger JM, Cheng H, Shibata A, Scholtz B, Schreiner S and Dunham N: The c-Fes family of protein-tyrosine kinases. Crit Rev Oncog 9: 43-62, 1998.

65. Greer P: Closing in on the biological functions of Fps/Fes and Fer. Nat Rev Mol Cell Biol 3: 278-289, 2002.

66. Haigh J, McVeigh J and Greer P: The fps/fes tyrosine kinase is expressed in myeloid, vascular endothelial, epithelial, and neuronal cells and is localized in the trans-golgi network. Cell Growth Differ 7: 931-944, 1996.

67. Rogers JA, Read RD, Li J, Peters KL and Smithgall TE: Autophosphorylation of the Fes tyrosine kinase. Evidence for an intermolecular mechanism involving two kinase domain tyrosine residues. J Biol Chem 271: 17519-17525, 1996. 
68. Read RD, Lionberger JM and Smithgall TE: Oligomerization of the Fes tyrosine kinase. Evidence for a coiled-coil domain in the unique N-terminal region. J Biol Chem 272:18498-18503, 1997.

69. Cheng HY, Schiavone AP and Smithgall TE: A point mutation in the N-terminal coiled-coil domain releases c-Fes tyrosine kinase activity and survival signaling in myeloid leukemia cells. Mol Cell Biol 21: 6170-6180, 2001.

70. Kanda S, Lerner EC, Tsuda S, Shono T, Kanetake H and Smithgall TE: The non-receptor protein-tyrosine kinase c-Fes is involved in fibroblast growth factor-2-induced chemotaxis of murine brain capillary endothelial cells. J Biol Chem 275: 10105-10111, 2000.

71. Kanda S, Landgren E, Ljungstrom M and Claesson-Welsh L: Fibroblast growth factor receptor 1-induced differentiation of endothelial cell line established from tsA58 large T transgenic mice. Cell Growth Differ 7: 383-395, 1996.

72. Eswarakumar VP, Lax I and Schlessinger J: Cellular signaling by fibroblast growth factor receptors. Cytokine Growth Factor Rev 16: 139-149, 2005.

73. Nakamura T, Mochizuki Y, Kanetake H and Kanda S: Signals via FGF receptor 2 regulate migration of endothelial cells. Biochem Biophys Res Commun 289: 801-806, 2001.

74. Senis Y, Zirngibl R, McVeigh J, Haman A, Hoang T and Greer PA: Targeted disruption of the murine fps/fes proto-oncogene reveals that $\mathrm{Fps} / \mathrm{Fes}$ kinase activity is dispensable for hematopoiesis. Mol Cell Biol 19: 7436-7446, 1999.

75. Zirngibl RA, Senis Y and Greer PA: Enhanced endotoxin sensitivity in fps/fes-null mice with minimal defects in hematopoietic homeostasis. Mol Cell Biol 22: 2472-2486, 2002.

76. Greer P, Haigh J, Mbamalu G, Khoo W, Bernstein A and Pawson T: The Fps/Fes protein-tyrosine kinase promotes angiogenesis in transgenic mice. Mol Cell Biol 14: 6755-6763, 1994.

77. Kontos CD, Stauffer TP, Yang WP, York JD, Huang L, Blanar MA, Meyer T and Peters KG: Tyrosine 1101 of Tie2 is the major site of association of p85 and is required for activation of phosphatidylinositol 3-kinase and Akt. Mol Cell Biol 18: 4131-4140, 1998.

78. Jones N, Master Z, Jones J, Bouchard D, Gunji Y, Sasaki H, Daly R, Alitalo K and Dumont DJ: Identification of Tek/Tie2 binding partners. Binding to a multifunctional docking site mediates cell survival and migration. J Biol Chem 274: $30896-$ 30905, 1999.

79. Kanda S, Miyata Y, Mochizuki Y and Kanetake H: Angiopoietin 1 is mitogenic for cultured endothelial cells. Cancer Res 65: 6820-6827, 2005

80. Kanda S, Mochizuki Y, Miyata Y and Kanetake H: The role of c-Fes in vascular endothelial growth factor-A-mediated signaling by endothelial cells. Biochem Biophys Res Commun 306: 1056-1063, 2003

81. Salcedo R and Oppenheim JJ: Role of chemokines in angiogenesis: CXCL12/SDF-1 and CXCR4 interaction, a key regulator of endothelial cell responses. Microcirculation 10: 359-370, 2003

82. Burger JA and Kipps TJ: CXCR4: a key receptor in the crosstalk between tumor cells and their microenvironment. Blood 107: 1761-1767, 2006

83. Tachibana K, Hirota S, Iizasa H, Yoshida H, Kawabata K, Kataoka Y, Kitamura Y, Matsushima K, Yoshida N, Nishikawa S, Kishimoto T and Nagasawa T: The chemokine receptor CXCR4 is essential for vascularization of the gastrointestinal tract. Nature 393: 591-594, 1998.
84. Kanda S, Mochizuki Y and Kanetake H: Stromal cell-derived factor-1alpha induces tube-like structure formation of endothelial cells through phosphoinositide 3-kinase. J Biol Chem 278: 257-262, 2003.

85. Pola R, Ling LE, Silver M, Corbley MJ, Kearney M, Blake Pepinsky R, Shapiro R, Taylor FR, Baker DP, Asahara T, Isner JM: The morphogen Sonic hedgehog is an indirect angiogenic agent upregulating two families of angiogenic growth factors. Nat Med 7: 706-711, 2001

86. Dyer MA, Farrington SM, Mohn D, Munday JR and Baron MH: Indian hedgehog activates hematopoiesis and vasculogenesis and can respecify prospective neurectodermal cell fate in the mouse embryo. Development 128: 1717-1730, 2001.

87. Byrd N, Becker S, Maye P, Narasimhaiah R, St-Jacques B, Zhang X, McMahon J, McMahon A and Grabel L: Hedgehog is required for murine yolk sac angiogenesis. Development 129: 361-372, 2002

88. Vokes SA, Yatskievych TA, Heimark RL, McMahon J, McMahon AP, Antin PB and Krieg PA: Hedgehog signaling is essential for endothelial tube formation during vasculogenesis. Development 131: 4371-4380, 2004.

89. Kanda S, Mochizuki Y, Suematsu T, Miyata Y, Nomata K and Kanetake H: Sonic hedgehog induces capillary morphogenesis by endothelial cells through phosphoinositide 3-kinase. J Biol Chem 278: 8244-8249, 2003 .

90. Condeelis J and Pollard JW: Macrophages: obligate partners for tumor cell migration, invasion, and metastasis. Cell 124: 263-266, 2006.

91. Knowles H, Leek R and Harris AL: Macrophage infiltration and angiogenesis in human malignancy. Novartis Found Symp 256: 189-200, 2004

92. Smithgall TE: Signal transduction pathways regulating hematopoietic differentiation. Pharmacol Rev 50: 1-19, 1998.

93. Rafii S, Lyden D, Benezra R, Hattori K and Heissig B: Vascular and haematopoietic stem cells: novel targets for anti-angiogenesis therapy? Nat Rev Cancer 2: 826-835, 2002.

94. Grunewald M, Avraham I, Dor Y, Bachar-Lustig E, Itin A, Jung S, Chimenti S, Landsman L, Abramovitch R and Keshet E: VEGFinduced adult neovascularization: recruitment, retention, and role of accessory cells. Cell 124: 175-189, 2006.

95. Uehara Y, Murakami Y, Mizuno S and Kawai S: Inhibition of transforming activity of tyrosine kinase oncogenes by herbimycin A. Virology 164: 294-298, 1988.

96. Bardelli A, Parsons DW, Silliman N, Ptak J, Szabo S, Saha S, Markowitz S, Willson JK, Parmigiani G, Kinzler KW, Vogelstein B and Velculescu VE: Mutational analysis of the tyrosine kinome in colorectal cancers. Science 300:949, 2003

97. Sangrar W, Zirgnibl RA, Gao Y, Muller WJ, Jia Z and Greer PA: An identity crisis for fps/fes: oncogene or tumor suppressor? Cancer Res 65: 3518-3522, 2005.

98. Delfino FJ, Stevenson H and Smithgall TE: A growth-suppressive function for the c-fes protein-tyrosine kinase in colorectal cancer. J Biol Chem 281: 8829-8835, 2006. 